\title{
Actitudes hacia la homosexualidad en adolescentes gallegos/as: aportaciones de la Pedagogía Queer
}

\section{Attitudes towards homosexuality in Galician adolescents: some contributions of Queer Pedagogy}

\author{
María Victoria Carrera-Fernández*, Ana Almeida**, Xosé Manuel Cid-Fernández*, Antonio González-Fernández*, \\ María Lameiras-Fernández*, Yolanda Rodríguez-Castro* \\ *Universidade de Vigo, Facultade de Ciencias da Educación de Ourense; ** Universidade do Minho, Instituto de Educação
}

\begin{abstract}
Resumen
El objetivo del trabajo es analizar las actitudes hacia la homosexualidad e identificar su relación con los estereotipos de género. Participaron 1405 adolescentes gallegos/as con una media de edad de 15.12 años. Se observa que los chicos son más instrumentales y expresan actitudes significativamente más negativas hacia la homosexualidad y el lesbianismo. Por otra parte, las chicas son más expresivas y manifiestan actitudes más positivas hacia la homosexualidad tanto femenina como masculina. $\mathrm{La}$ instrumentalidad correlaciona positivamente con la homofobia y la expresividad correlaciona negativamente tanto con la homofobia como con la lesbofobia. Se destacan las aportaciones de la Pedagogía Queer.

Palabras clave: adolescencia, homofobia, estereotipos de género, Pedagogía Queer.
\end{abstract}

\begin{abstract}
The aim of this study is to analyze attitudes towards homosexuality and to identify their relationship with gender stereotypes. A total of 1405 Galician adolescents (mean age $=15.12$ years) participated in the study. Boys are more instrumental and exhibite more negative attitudes towards homosexuality and lesbianism than girls. By constrast, girls exhibite more favorable attitudes towards both female and male homosexuality. Instrumentality correlates positively with homophobia, and expressiveness correlates negatively with both homophobia and lesbophobia. Contributions of Queer Pedagogogy are discussed.

Keywords: adolescence, homophobia, gender estereotypes, Queer Pedagogy.
\end{abstract}

\section{Introducción}

El término homofobia fue acuñado en 1971 por Smith, que lo definió como una actitud de miedo y rechazo a la homosexualidad y que implica, por tanto, una actitud discriminatoria dirigida hacia una persona en función de su identidad homosexual. Se caracteriza por un sentimiento de superioridad con respecto al otro, deshumanización del otro, que se ve como carente de emociones y conciencia, y convicción de ser merecedor de privilegios y estar en la posición correcta (Viñuales, 2002).

Una compleja definición de homofobia sería el miedo, de los hombres a amar a otros hombres, de hecho, la identidad masculina tradicional se construye sobre la misoginia y la homofobia (Epstein, O’Flynn, y Teldford,
2003). Desde esta perspectiva, la homofobia no sólo afecta a los homosexuales, sino a todos los hombres que no se adaptan a los estereotipos tradicionales impuestos para su género, afectando a todos ellos sin excepción, con la amenaza de degradarles al estatus de "maricas", "calzonazos" o "nenazas" (Guasch, 2006). Poniéndose de relieve la rigidez de la matriz heterosexual (Butler, 1990, 1993), de modo que transgredir cualquier estereotipo de género relativo a la masculinidad tradicional pone en cuestionamiento la heterosexualidad.

Asimismo, la homofobia, en tanto que actitud discriminatoria dirigida hacia una persona en función de su identidad homosexual, va dirigida también hacia las mujeres lesbianas, lo que se conoce también como lesbofobia (Viñuales, 2002), término que pone de manifiesto la doble discriminación a la que está sujeta la mujer lesbiana en la cultura occidental: por ser mujer y por ser lesbiana, lo que supone un prejuicio añadido, con las consecuentes repercusiones en la calidad de vida de estas mujeres.

Las actitudes homófobas abarcan desde las actitudes discriminatorias más hostiles y explícitas hasta aquellas otras que, de una forma latente y sutil, permanecen silenciadas e invisibles. En este sentido, podríamos afirmar que existen actitudes homófobas explícitas y hostiles, tales como la agresión verbal, física o psicológica hacia las personas homosexuales; y actitudes homófobas latentes o sutiles (Quiles, Betancor, Rodríguez, Rodríguez y Coello, 2003), tales como los discursos culturales o médicos sobre el carácter patológico de la homosexualidad o la incapacitación de las parejas homosexuales para la crianza, entre otras cuestiones que pretenden coartar la vivencia y expresión homosexual. Actitudes que constituyen lo que Lizárraga (2005) denomina "violencia polimórfica" y Eribon (2000) "discursos culturales" y "científicos", desarrollados por la ciencia y argumentados en los medios de comunicación, la universidad o el congreso. De esta forma la homofobia no es sólo el insulto grosero o explícito en la calle, sino que constituye, tal y como destaca Lizárraga (2005, p.33), "una persistente presencia fantasmal que sobrevuela la cotidianidad sembrando temores y resentimientos, insidias, culpas, vergüenzas y desconfianzas..."

En esta línea Raja y Stokes (1998) desarrollaron la escala de Homofobia Moderna para analizar las actitudes 
de rechazo más sutiles hacia homosexuales y lesbianas. Incluyendo tres dimensiones que hacen referencia al malestar personal, entendido como la incomodidad que genera la presencia de homosexuales y lesbianas, y la necesidad de evitar el contacto personal con este colectivo; a la homofobia institucional, referida a las actitudes hacia las prácticas sistemáticas de exclusión al colectivo de gays y lesbianas que se despliegan desde el ámbito gubernamental, laboral o religioso; y al carácter desviado y modificable de estas orientaciones sexuales, referida a la creencia de que la homosexualidad y el lesbianismo son enfermedades o perversiones que pueden ser curadas y modificadas.

Casi medio siglo después de las revueltas de Stonewall los avances en relación a la diversidad sexual son innegables en prácticamente todas las sociedades que se consideran a sí mismas desarrolladas. Sin embargo, no podemos afirmar que occidente constituya un oasis de libertades y derechos para el colectivo de Lesbianas, Gays, Bisexuales, Transexuales, Intersexuales, Queer y Questioning (LGBTIQQ), sino más bien un espejismo. Así, tal y como ponen de manifiesto las últimas macroencuestas internacionales, el rechazo a la diversidad sexual continúa siendo un gran mal de múltiples caras, desde algunas más hostiles y explícitas hasta otras aparentemente más benévolas y silenciosas, que nos recorre de norte a sur y de este a oeste (European Union Agency for Fundamental Righs -FRA-, 2014, 2016; Kann et al., 2016).

En EE.UU los resultados de una encuesta nacional llevada a cabo en 25 estados en 2015 (Kann et al., 2016), pone de relieve que un $60.4 \%$ de los/as adolescentes LGBTIQQ frente al $26.4 \%$ de sus iguales que reproducen la norma de género se han sentido tristes casi todos los días durante las últimas 2 o más semanas. En esta línea, el $42.8 \%$ frente al $14.8 \%$ ha considerado seriamente suicidarse en el último año. Asimismo, los datos revelan que sufren más significativamente situaciones de violencia sexual, así como de bullying en el ámbito escolar, inseguridad en la escuela o en el camino a la escuela que provoca absentismo.

Paralelamente, en Europa se lleva a cabo una encuesta sobre la situación de las personas LGBTQQ en la Unión Europea en el año 2012, que constituye la mayor recopilación de este tipo disponible en Europa y en todo el mundo hasta la fecha (FRA, 2014). Entre los resultados obtenidos se comprueba que el $70 \%$ de las personas tanto españolas como portuguesas entrevistadas ha sufrido comentarios o comportamientos negativos en la escuela por su identidad sexual, y en torno al $60 \%$ ha escondido o disfrazado su identidad durante su escolaridad. Concluyendo que la discriminación que sufre el colectivo LGBTIQQ es más la norma que la excepción.

En la misma línea, estudios realizados en España y Portugal señalan que alrededor del $50 \%$ de los adolescentes y jóvenes LGBTIQQ han sufrido bullying sistemáticamente (Antonio y Moleiro, 2015; DevísDevís et al., 2017; Federación Estatal de Lesbianas, Gais, Transexuales y Bisexuales, 2012; Rodrigues, Gravea, Oliveira, y Nogueira, 2016)). La ideación suicida y el intento de suicidio en adolescentes y jóvenes españoles y portugueses se han relacionado consistentemente con estas situaciones (Baiocco et al., 2014; Pereira y Rodrigues, 2015), así como la homofobia internalizada (Pereira y Rodrigues, 2015).

La situación del colectivo LGBTIQQ es, por tanto, preocupante, pero no sorprendente si tenemos en cuenta las actitudes sociales hacia estos colectivos, que distan mucho de ser positivas. Concretamente, los estudios llevados a cabo con adolescentes y jóvenes españoles y portugueses comprueban que las actitudes hacia el colectivo LGBTIQQ en ambos países son negativas (Carrera, Lameiras, Rodríguez y Vallejo, 2014; Costa y Davies, 2012; Lopes, Oliveira, Nogueira y Grave, 2017; Rodríguez, Lameiras, Carrera y Vallejo, 2013); especialmente en los adolescentes y jóvenes varones (Carrera et al., 2014; Costa y Davies, 2012; Lopes et al., 2017; Rodríguez et al., 2013) católicos, con ideología de derechas y con pocos amigos/as no heterosexuales (Lopes et al., 2017).

Las anteriores premisas ponen de relieve que el estudio de las actitudes hacia la homosexualidad en adolescentes es clave para comprender la influencia de los diferentes agentes de socialización, incluida la escuela, en la tolerancia hacia la diversidad sexual y, especialmente, para llevar a cabo las estrategias de intervención educativa que permitan el desarrollo de actitudes positivas hacia la diversidad sexual.

\section{Método}

\section{Participantes}

Una muestra formada por 1405 adolescentes de la Comunidad Autónoma Gallega, escolarizados en los dos últimos cursos de Educación Secundaria Obligatoria, participó en el estudio. El 54.5\% son chicas y el $45.5 \%$ chicos con una media de edad de 15.12 años $(D T=.97)$ y un rango de 13 a 19 años.

En relación a su origen cultural/étnico el $82.6 \%$ se considera español/a, el $9.5 \%$ gallego/a, el $3.5 \%$ sudamericano/a, el $0.9 \%$ gitano/a, el $0.5 \%$ africano, el $0.1 \%$ asiático, y un $2.9 \%$ se identifica con otras categorías de identidad. En cuanto al nivel cultural, el $33.2 \%$ de las madres del alumnado tiene estudios secundarios postobligatorios, el $29.3 \%$ estudios universitarios, el $25.6 \%$ estudios secundarios, el $10.8 \%$ posee exclusivamente estudios primarios y el $1.1 \%$ no tiene estudios. Identificándose una distribución similar también para los padres $(32.7 \%$ posee estudios secundarios postobligatorios, $28 \%$ estudios secundarios obligatorios, $24.1 \%$ completó estudios universitarios, $13.5 \%$ posee estudios primarios y $1.6 \%$ no tiene estudios)

\section{Instrumentos}

Se administró un cuestionario en el que se incluían variables sociodemográficas, así como las siguientes escalas:

Escala de Homofobia Moderna (Modern Homophobia Scale, Raja y Stokes, 1998; version Española de Rodríguez et al., 2013). Se administró la subescala de malestar interpersonal hacia gays y lesbianas, constituida a su vez por la subescala de Actitudes hacia homosexuales (por ejemplo: "Estoy abierto/a a nuevos 
amigos homosexuales"), con un total de 9 ítems ( $\alpha=.91)$; y la subescala de Actitudes hacia lesbianas (por ejemplo: "No me importaría ir a una fiesta a la que asistan lesbianas"), con un total de 10 ítems $(\alpha=.90)$. Para contestar se presenta un formato de respuesta tipo Likert de 1 (Totalmente en desacuerdo) a 6 (Totalmente de acuerdo) en el que a menores puntuaciones actitudes más negativas hacia gays y lesbianas.

Escala de expresividad (Femininity Trait Index, FTI, Barak y Stern, 1986). Esta escala mide la autoadscripción de cada persona a rasgos de personalidad expresivos, tradicionalmente considerados como femeninos (por ejemplo: "cariñoso/a"). Está formada por 10 ítems con un formato de respuesta tipo Likert de 1 (nunca me describe) to 6 (siempre me describe) en el que a mayores puntuaciones mayor adscripción a rasgos expresivos $(\alpha$ $=.90)$.

Escala de instrumentalidad (Masculinity Trait Index, MTI, Barak y Stern, 1986). Esta escala mide la autoadscripción de cada persona a rasgos de personalidad instrumentales, tradicionalmente considerados como masculinos (por ejemplo: "competitivo/a"). Está formada por 10 ítems con un formato de respuesta tipo Likert de 1 (nunca me describe) to 6 (siempre me describe) en el que a mayores puntuaciones mayor adscripción a rasgos instrumentales $(\alpha=.86)$.

\section{Procedimiento}

Se llevó a cabo un estudio correlacional a través de encuesta por muestreo aleatorio y de carácter transversal durante el curso escolar 2012-2013.

Se envió una carta a los/las directores/as de los centros educativos seleccionados presentado el estudio y pidiendo su colaboración; y posteriormente se contactó telefónicamente para confirmar su participación. En cada escuela participante se solicitó consentimiento informado activo de los padres/madres o tutores/as legales, excluyendo del estudio a todo el alumnado que no obtuvo la autorización.

El cuestionario fue de carácter auto-administrado, voluntario y anónimo, y fue administrado en horario lectivo entre los meses de marzo y junio de 2013 por dos investigadoras con formación psicopedagógica y especialmente formadas para esta tarea. El alumnado disponía de aproximadamente 20 minutos para cubrir el cuestionario.

\section{Resultados}

Tal y como puede observarse en la Tabla 1, los chicos expresan actitudes significativamente más negativas que sus compañeras tanto hacia la homosexualidad femenina como hacia la homosexualidad masculina, especialmente hacia esta última. Asimismo, se observa que ellos son significativamente más instrumentales y menos expresivos. Por su parte, las chicas manifiestan actitudes significativamente más positivas hacia la homosexualidad tanto femenina como masculina, y son significativamente más expresivas y menos instrumentales.
Tabla 1

Diferencias en función del sexo en las escalas analizadas

\begin{tabular}{|c|c|c|c|}
\hline & & Media $(D T)$ & Diferencias \\
\hline HG & $\begin{array}{l}\text { Chicas } \\
\text { Chicos }\end{array}$ & $\begin{array}{c}5.54(.73) \\
4.35(1.28)\end{array}$ & $t(1405)=20.12^{* * *}$ \\
\hline HL & $\begin{array}{l}\text { Chicas } \\
\text { Chicos }\end{array}$ & $\begin{array}{c}5.36(.85) \\
5.05(1.03)\end{array}$ & $t(1405)=5.96 * * *$ \\
\hline $\mathrm{E}$ & $\begin{array}{l}\text { Chicas } \\
\text { Chicos }\end{array}$ & $\begin{array}{l}5.05(.71) \\
4.65(.96)\end{array}$ & $t(1405)=8.53 * * *$ \\
\hline I & $\begin{array}{l}\text { Chicas } \\
\text { Chicos }\end{array}$ & $\begin{array}{l}3.63(.98) \\
4.24(.99)\end{array}$ & $t(1405)=-11.16^{* * *}$ \\
\hline
\end{tabular}

Homofobia hacia lesbianas; $\mathrm{E}=$ Expresividad; $\mathrm{I}=$ Instrumentalidad.

Por lo que respecta a las correlaciones entre las escalas analizadas se comprueba que la instrumentalidad correlaciona moderada y positivamente con la homofobia hacia gays, y la expresividad correlaciona moderada y negativamente tanto con la homofobia hacia gays como con la homofobia hacia lesbianas.

Tabla 2

Correlaciones de Pearson entre las escalas analizadas

\begin{tabular}{lcccc} 
& HG & HL & E & I \\
\hline HG & & $.61^{* *}$ & $.23^{* *}$ & $-.24^{* *}$ \\
HL & & & $.18^{* *}$ & .03 \\
E & & & & $.08^{* *}$
\end{tabular}

I

Nota. $* * p<.01 ; * * p<.001 ; \mathrm{HG}=$ Homofobia hacia Gays; $\mathrm{HL}=$ Homofobia hacia lesbianas; $\mathrm{E}=$ Expresividad; $\mathrm{I}=$ Instrumentalidad.

\section{Discusión}

En este trabajo se han analizado las actitudes hacia la homosexualidad masculina y femenina en un grupo de adolescentes gallegos/as, así como las relaciones entre la homofobia y los estereotipos de género tradicionalmente masculinos -dimensión instrumental- y femeninos dimensión expresiva.

Los resultados ponen de relieve que los chicos son más instrumentales y expresan actitudes significativamente más negativas hacia la homosexualidad y el lesbianismo. Por otra parte, las chicas son más expresivas y manifiestan actitudes más positivas hacia la homosexualidad tanto femenina como masculina. Asimismo, se observa que la instrumentalidad correlaciona moderada y positivamente con la homofobia, y la expresividad correlaciona en sentido contrario, es decir moderada y negativamente, tanto con la homofobia como con la lesbofobia.

Las actitudes significativamente más negativas de los chicos hacia los homosexuales y las lesbianas están en la línea de los estudios sobre esta problemática llevados a cabo con muestras tanto de adolescentes (Carrera et al, 2014; Costa y Davies, 2012; Rodríguez, 2013) como de población adulta (Lopes et al., 2017; Raja y Stokes, 1998)

El mayor rechazo de los chicos hacia la transgresión del modelo heteronormativo podría estar influenciado por la mayor rigidez del modelo de socialización 
masculina (Connell, 2000), ya que, tal y como señalan Epstein et al. (2003), la identidad masculina tradicional se construye sobre la misoginia y la homofobia.

Por otra parte, el hecho de que los y las adolescentes que se describen a sí mismos/as con estereotipos expresivos -tradicionalmente considerados como femeninos- sean también los/as que tienen actitudes más positivas hacia homosexuales y lesbianas, contrariamente a lo que sucede con los estereotipos de género instrumentales -tradicionalmente relacionados con la masculinidad hegemónica-, pone de relieve que, para fomentar actitudes positivas hacia la diversidad sexual, es necesario promocionar el desarrollo integral de la personalidad, libre de roles y estereotipos de género, apostando por valores expresivos tanto para chicos como para chicas, basados en la empatía, la afectividad, el cuidado, la sensibilidad y la solidaridad con el otro. Apostar por una socialización igualitaria basada en la ética del cuidado y el desarrollo de la inteligencia emocional es una herramienta crucial no sólo para la promoción de actitudes positivas hacia la diversidad sexual, sino también para la prevención de las consecuencias negativas que se derivan del rechazo a la diversidad, tales como el bullying o los delitos de odio.

Especialmente útil para el ejercicio de una práctica educativa liberadora es la Pedagogía Queer. Basada en la teoría filosófica de Butler (1990, 1993) la Pedagogía Queer denuncia la violencia que genera la socialización diferencial de género heteronormativa, y apuesta por la ruptura de las normas de género y la valoración de las identidades sexuales diversas. Los ejes definitorios de esta pedagogía se organizan en torno a la necesidad de "pensar el mundo" más allá del rígido binomio hombremasculino-heterosexual/mujer-femenina-heterosexual, rompiendo la imposición de una identidad unitaria, fija y coherente que, como destaca Butler (1993), constituye el "lecho" sobre el que se erige la violencia y la exclusión de las identidades que son percibidas como ininteligibles, abyectas o amenazantes para la coherencia del sujeto, imposibilitando toda forma de vida situada más allá del marco heteronormativo.

Una pedagogía que a través de la educación de las sexualidades y en las sexualidades, posibilite aquellas estrategias necesarias para desarrollar formas más confortables de organizar la identidad (Epstein et al., 2003) y que contribuya a la construcción de sociedades más justas y democráticas para todos y todas.

\section{Referencias}

António, R., Pinto, T., Pereira, C., Farcas, D., y Moleiro (2012). Bullying homofóbico no contexto escolar em Portugal. Psicologia, 26(1), 17-32.

Baiocco, R., Loverno, S., Cerutti, R., Santamaria, F., Fontanesi, L., Lingiardi, V., Baumgartner, E., y Laghi, E. (2014). Suicidal ideation in Spanish and Italian lesbian and gay young adults: The role of internalized sexual stigma. Psicothema, 26(4), 490-496. doi: 10.7334/psicothema2014.1

Barak, S., y Stern, B. (1986). Sex-linked trait indexes among baby-boomeers and pre-boomers. A research note. In R. Lutz (Ed.), Advances in consumer research (pp. 53-68). Provo: Association for Consumer Research.

Butler, J. (1990). Gender trouble: feminism and the subversion of identity. London and New York: Routledge.

Butler, J. (1993). Bodies that Matter: On the Discursive Limits of "Sex". London and New York: Routledge.

Carrera-Fernández, M.V., Lameiras-Fernández, M., Rodríguez-Castro, Y., y Vallejo-Medina, P. (2014). Spanish adolescents' attitudes toward transpeople: proposal and validation of a short form of the Genderism and Transphobia Scale. Journal of Sex Research, 51(6), 654-666. doi: 10.1080/00224499.2013.773577

Connell, R.W. (2000). The men and the boys. Berkeley: University of California Press.

Costa, P.A., y Davies, M.D. (2012). Portuguese adolescents' attitudes toward sexual minorities: Transphobia, homophobia, and gender role beliefs. Journal of Homosexuality, 59(10), 1424-1442. doi:10.1080/00918369.2012.724944

Devís-Devís, J., Pereira-García, S., Valencia-Peris, A., Fuentes-Miguel, J., López-Cañada, E., \& PérezSamaniego, V. (2017). Harassment patterns and risk profile in Spanish trans persons. Journal of Homosexuality, 64(2), 239-255. doi: 10.1080/00918369.2016.1179027

Epstein, D., O'Flynn, S. y Teldford, D. (2003). Silenced sexualities in schools and universities. Stoke-on-Trent: Trenthan Books.

Eribon, D. (2000). Identidades. Reflexiones sobre la cuestión gay. Barcelona: Bellaterra.

European Union Agency for Fundamental Rights (2014) European Union lesbian, gay, bisexual and transgender survey. Recuperado de http://goo.gl/mb4YDF.

Federación Estatal de Lesbianas, Gais, Transexuales y Bisexuales (2012). Acoso escolar homofóbico y riesgo de suicidio. Recuperado de http://goo.gl/TsnEIT

Guasch, O. (2006). Héroes, científicos, heterosexuales y gays. Los varones en perspectiva de género. Barcelona: Bellaterra.

Kann, L., Olsen, E. O., McManus, T., et al. (2016). Sexual Identity, Sex of Sexual Contacts, and HealthRelated Behaviors Among Students in Grades 9-12 United States and Selected Sites, 2015. Recuperado de https://www.cdc.gov/mmwr/volumes/65/ss/ss6509a1. htm

Lizárraga, A. (2005). Homofobia: enfermedad y germen. Revista de Estudios de Antropología Sexual, 1(1), 3342.

Lopes, D., Oliveira, J. M., Nogueira, C., y Grave, R. (2017). The social determinants of polymorphous prejudice against lesbian and gay individuals: the case of Portugal. Sexuality Research and Social Policy, 14(1), 56-70. doi:10.1007/s13178-016-0230-4

Pereira, H., y Rodrigues, P. (2015). Internalized homophobia and suicidal ideation among LGB youth. Journal of Psychiatry, 18(2),1-6. doi: 10.4172/Psychiatry.1000229 
Quiles, M., Betancor, V., Rodríguez, R., Rodríguez, A. y Coello, E. (2003). La medida de la homofobia manifiesta y sutil. Psicothema, 15, 197-204.

Raja, S. H., y Stokes, J. P. (1998). Assessing attitudes toward lesbians and gay men: The Modern Homophobia Scale. Journal of Gay, Lesbian and Bisexual Identity, 3, 113-134.

Rodrigues, L., Gravea, R., Oliveira, J.M., y Nogueira, C. (2016). Study on homophobic bullying in Portugal using Multiple Correspondence Analysis (MCA). Revista Latinoamericana de Psicología, 48, 191-200. doi: 10.1016/j.rlp.2016.04.001

Rodríguez, Y., Lameiras, M., Carrera, M. V., y Vallejo, P. (2013). Validación de la Escala Moderna de Homofobia en una muestra de adolescentes. Anales de Psicología, 29(2), 523-533. doi: 10.6018/analesps.29.2.137931

Smith, K. T. (1971). Homophobia: A tentative personality profile. Psychological Reports, 29, 10911094.

Viñuales, O. (2002). Lesbofobia. Barcelona: Bellaterra. 